\title{
Active thermography as a tool to investigate heat and gas transfer across the air- water interface
}

\author{
by J. Kunz ${ }^{1,2}$ and B. Jähne ${ }^{1,2}$ \\ 1) Institute of Environmental Physics, Heidelberg University, Im Neuenheimer Feld 229, 69120 Heidelberg, \\ Germany, jakob.kunz@iup.uni-heidelberg.de \\ 2) Heidelberg Collaboratory for Image Processing, Berliner Straße 43, 69120 Heidelberg, Germany, \\ bernd.jaehne@iwr.uni-heidelberg.de
}

\begin{abstract}
Gas and heat exchange between the ocean and the atmosphere are a key process in the earth system. Active thermography is a tool that can be used to estimate heat and gas transfer rates with unprecedented temporal and spatial resolution compared to other measurement techniques. In this paper recent technical progress of this method is described: a) a spatially more homogeneous heating of the water by a carbon dioxide laser for measurements with a significant lower systematic error and statistical uncertainty, and b) a new modulation scheme for faster measurements. While the latter is essential for field measurements, the first enables the distinction of different models for air-water gas exchange in the laboratory.
\end{abstract}

\section{Introduction}

The exchange of gases and heat between the ocean and the atmosphere has tremendous importance for the earth's climate. Due to the increasing amount of carbon dioxide that we emit into the atmosphere we increase gas fluxes of $\mathrm{CO}_{2}$ into the ocean. However, these fluxes are difficult to measure precisely and even harder to parametrize by a model. Nevertheless, accurate climate models need to incorporate how fast and how much $\mathrm{CO}_{2}$ and other climate relevant gases leave the atmosphere and go into the oceans.

The study of air-sea gas exchange has a history of about 30 years. Still no accurate model or parametrization could be derived from the various measurements that have been carried out over this time period. This is due to the complex turbulent processes that drive gas exchange.

The exchange is mainly driven by the wind that acts on the water surface. Accordingly surface active material strongly influences the exchange process by reducing the surface tension and thus modifying the wave creation by the wind and altering the amount of turbulence present directly at the air-water interface.

To quantify the transport rate, the so called transfer velocity $\mathrm{k}$ is used. It is the proportionality constant between the concentration difference $\Delta c$ of a certain trace gas in air and water respectively and the flux $j$ of this trace gas through the air-water interface:

$$
k_{\text {gas }}=\frac{j_{\text {gas }}}{c_{\text {air }}-c_{\text {water }}}=\frac{j_{\text {gas }}}{\Delta c}
$$

In a typical gas exchange experiment, one needs to monitor the gas concentration of a trace gas in the air, respectively water for a certain amount of time. The flux between the air and water compartments can then be estimated from the concentration changes over time. However, it is often necessary to add trace gases artificially in order to get large concentration changes that can be measured accurately. And this of course is a difficult undertaking on the ocean. If for example the concentration of a certain trace gas in the air is monitored, a decrease in this concentration can be a result of the transport into the ocean, but it can also simply mean that the gas has been diluted by the wind in the atmosphere and has been spread over a larger volume.

There are some advanced techniques like the dual tracer technique $[1,2]$, where not the absolute concentration of a trace gas, but the ratio of two different trace gases is monitored and analyzed. But the exchange rates of suitable tracers are slow and this leads to long measurement times. Another approach is to use radioactive tracers $[3,4]$. But then the detection is limited by the decay time of the available tracer. So both of these methods suffer from long measurement times and this is highly critical for precise measurements, as boundary conditions like wind speeds on the ocean will hardly remain constant for a long measurement.

The exchange of heat is driven by the same mechanisms as the exchange of gases. This similarity makes heat a very versatile tool for the investigation of small scale air-sea exchange processes. The investigation of heat exchange can be done very efficiently via thermography, which is a non-contact technique that does not interfere with the turbulent processes at the air-water interface.

Thermography can be used to observe surface turbulence directly, either actively by heating local structures on the water surface and monitoring the evolution of these structures or by passively monitoring the temperature changes 


\subsection{1/qirt.2016.069}

that are due to evaporation and water mixing at the water surface. In this way it is for example possible to derive surface drift velocities [5]. If the gas exchange process is also made visible and detectable for cameras by a fluorescent dye and suitable light sources, then thermography together with cameras that operate in the visible wavelength regime, can be used to get a visual comparison of gas and heat exchange processes at the air-water interface $[6,7]$.

However, thermography can not only be used to visualize heat exchange, but also to derive heat transfer velocities [8-13]. As the driving mechanisms for heat and gas exchange are the same, it is possible to scale to gas transfer velocities from measured heat transfer velocities and therefore indirectly measure gas transfer velocities [13]. This is especially important in cases, where it is otherwise very difficult to get gas transfer rates, e.g. during field experiments on a ship. The scaling relies on the Schmidt number Sc of heat and the trace gas and on the Schmidt number exponent $n$, which is $1 / 2$ for a rough water surface and changes to $2 / 3$ when going to a smooth water surface:

$$
\frac{k_{1}}{k_{2}}=\left(\frac{S c_{1}}{S c_{2}}\right)^{-n}
$$

It should be mentioned, that all thermographic measurement approaches have significantly shorter measurement times than the gas exchange measurements, ranging between several minutes to an hour compared to time spans for the gas exchange measurements of several hours to several days.

The next section will first describe the evolution of the measurement system starting with the first apparatus that was used in the 1980s. Then the experimental setup will be described, followed by a description of the beam shaping. Afterwards the excitation scheme is explained and a new approach to reduce the measurement time is presented.

\section{Measurement technique}

The main idea of the active thermography technique is to locally heat an area on the water surface and to monitor the temperature change at the water surface. This is similar to adding a trace gas and to monitor its concentration change, however as only a local area is heated, only this area takes part in the exchange process, while for a trace gas the global surrounding of the gas will influence the measurement.

\subsection{Earlier measurement techniques}

Heat transfer velocities across the aqueous viscous boundary layer can be measured actively by the use of a heat source $[8,9,10,13]$ or passively by exploiting the effect of surface cooling due to evaporation $[11,12]$. The first measurements by Jähne et al. [8] were done using active thermography. The basic concept of the measurement was the same like in our present setup and will be discussed in detail in section 2 . However, the early setup could only measure at one point on the water surface with an IR radiometer, as no infrared cameras were available back then. The heat source was an IR radiator that could not be switched on and off quickly. In order to be able to do a periodic excitation (details are given in section 2.3) a chopper was needed to modulate the heating. This made it impossible to apply modulation frequencies higher than $2 \mathrm{~Hz}$.

One of the biggest improvements for the investigation of heat transfer was the use of infrared cameras. In 1995 a new measurement technique had been introduced $[9,10]$. The temperature was measured with an infrared camera and as a heat source a $25 \mathrm{~W} \mathrm{CO}$ laser was used. This measurement technique did not only differ in the experimental components used for heating the water and detecting the temperature changes, but it also applied a new method to probe the air-water interface. Instead of heating the water surface periodically, now single dots were printed onto the water surface, recorded by the IR camera and tracked with methods from image processing. From numerical simulations a model has been derived that could be used to fit the measured temperature decay. From this fit the heat transfer velocity could be obtained.

A few years later another approach was tested to estimate heat transfer velocities [11]. This time no artificial heat source was used, but the natural temperature changes at the water surface that arise from evaporation and mixing of surface and bulk water were used. The heat distribution at the water surface can be detected with an IR camera. The use of a model together with a statistical analysis of the temperature distribution allowed to estimate heat transfer velocities.

The problem with the latter two techniques is, that they rely on a model. There have been controversies, especially with the active measurement technique where dots are tracked, when differences between directly measured gas transfer velocities and heat transfer velocities that have been scaled to gas transfer velocities via equation 2 have been observed [14].

This is why the old measurement approach from [8] came up again, as this is the only technique that does not rely on model assumptions. The technological advancement has made IR cameras and strong compact $\mathrm{CO}_{2}$ lasers available and replaced the old IR radiometer and the IR radiator. As lasers can operate at high frequencies, the measurements could make use of a broader excitation frequency range. With this new setup it could be shown, that without model assumptions involved the scaling from heat to gas transfer rates seems possible [13].

Still there was one significant drawback in the new measurement setup. The output profile of a $\mathrm{CO}_{2}$ laser is a small circle with a Gaussian intensity profile. To avoid the effect of horizontal diffusion, it is necessary to heat an area 


\subsection{1/qirt.2016.069}

and thus beam shaping was necessary. Therefore, the beam was spread into a laser sheet with a cylindrical lens in one dimension and then this laser sheet was moved forwards and backwards along the other dimension in order to heat a rectangular area. The resulting intensity profile was a rectangular area with a Gaussian intensity distribution along the cross-scanning direction. As a result, the heat flux at the water surface was not homogeneous. Water flowing through the heated area will therefore be heated with the mean heat flux, which depends on the actual path the water takes when travelling through the illuminated area. This path is hard to reconstruct and thus the effective heat flux is unknown.

To overcome this problem, the latest change in the experimental setup was to alter the way of beam shaping. Instead of a moving mirror we are now using a diffractive beam homogenizer (Holo/Or HM-271-A-Y-A and Holo/Or HH211-A-Y-A). This device shapes the incoming beam into a square intensity profile with a very good homogeneity. Due to the diffractive operation principle there is an intensity spike in the middle of the output profile from the $0^{\text {th }}$ diffraction order, but this is not a problem for our measurements. More details about the beam shaping can be found in section 2.3 .

\subsection{Experimental setup}

One or two $100 \mathrm{~W} \mathrm{CO}_{2}$ lasers (Synrad Evolution 100, Iradion Infinity 100) operating at $10.6 \mu \mathrm{m}$ wavelength are used to heat the water surface. This wavelength is ideal for our application, as on the one hand nearly no radiation is absorbed in the air space and on the other hand water has such a high absorption coefficient at this wavelength that practically all of the radiation is absorbed within the topmost $20 \mu \mathrm{m}$ of the water surface. Thus the $\mathrm{CO}_{2}$ lasers represent the ideal tool to precisely deposit energy into the top layer of the water surface.

To measure the temperature changes at the water surface, we use an infrared camera (IRCAM Velox 327k M) that operates in the 3 to $5 \mu \mathrm{m}$ wavelength range with an NETD of $20 \mathrm{mK}$ and a resolution of $640 \times 512$ pixels. By separating the wavelength to heat the water surface from the wavelength range to measure the temperature any influence of laser reflections from the water surface on the temperature measurements is avoided. This was not possible with the early setup of Jähne et al. [8].

A schematic sketch of our experimental setup as used in our laboratory in Heidelberg can be seen in figure 1.

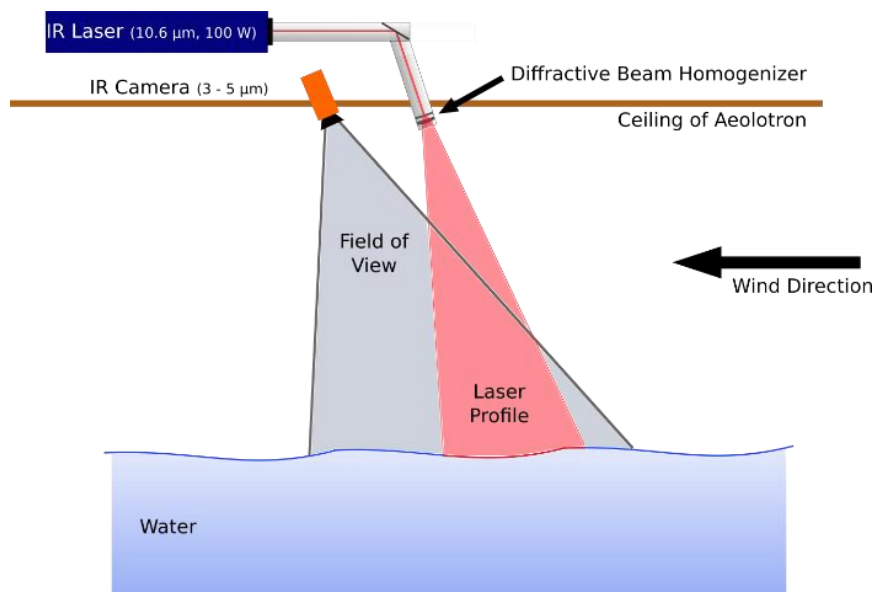

Fig. 1. Schematic drawing of the experimental setup in the Heidelberg wind-wave tank Aeolotron.

\subsection{Heating an area}

We need to heat a sufficiently large area on the water surface. There are two reasons for this. The first one is, that we want to have an area large enough to be able to perform our analysis only on an inner part of the heated area, because then we don't need to consider horizontal diffusion, as the surrounding water of this inner part has the same temperature.

The second reason is, that the water needs a certain time within the heated area to reach a thermal equilibrium between the incoming energy from the laser radiation and outgoing of energy by the transport of heat into the bulk water. Figure 2 visualizes the heating process of the water as it flows through the illuminated area. The part that is labelled "thermal equilibrium" is the area that is used for the data analysis. 


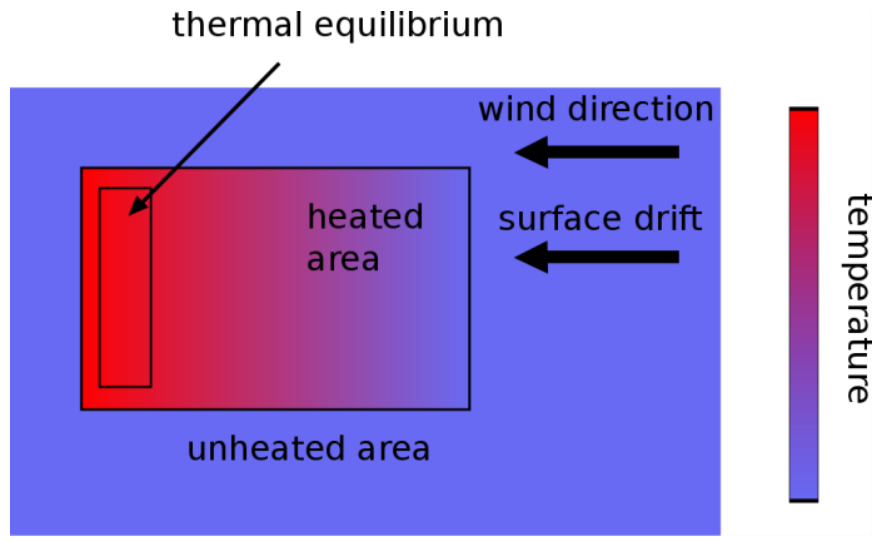

Fig. 2. Illustration of the heating process. The water flows from the right to the left and crosses the area that is illuminated by the $\mathrm{CO}_{2}$ laser. While it flows through the illuminated area the water heats up, unit it reaches a stable temperature.

As we want to have a constant heat flux at the water surface we need a homogeneous laser intensity profile. To achieve this, we use a diffractive beam homogenizer. This device shapes the incoming beam into a square intensity profile. Due to the design of the element the $0^{\text {th }}$ diffraction order cannot be suppressed completely and thus an intensity spike remains in the middle of the homogenized area. But apart from this spike there is a broad intensity plateau. This is a huge advantage compared to an older setup where the laser beam had been broadened and moved back and forth with a moving mirror to heat an area. The solution with the moving mirror suffered from the remaining Gaussian profile and thus from an inhomogeneous intensity profile. The intensity profiles of both setups are shown in figure 3 for comparison. Additionally, figure 8 shows the improved overall measurement accuracy due to the more homogeneous heat flux at the water surface.
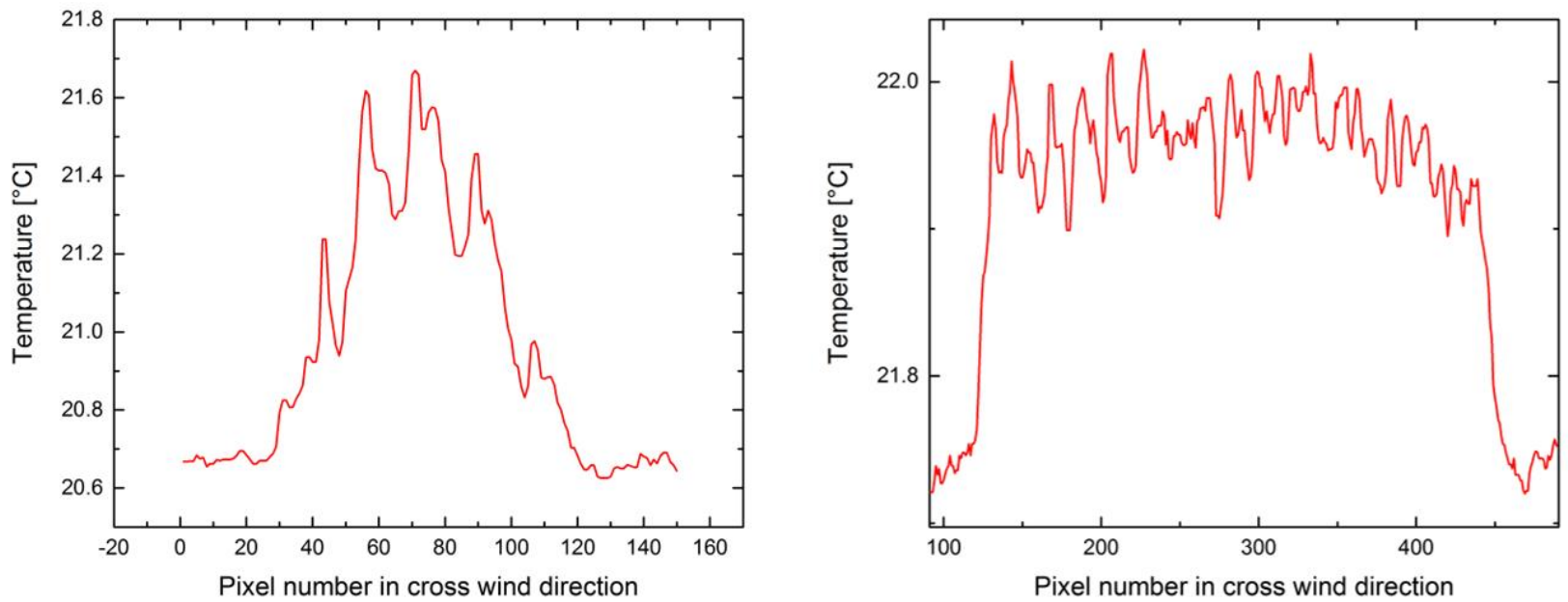

Fig. 3. Comparison of profiles of the intensity distribution at the water surface for two different methods of beam shaping. The left figure shows the profile of a laser beam that has been broadened with a cylindrical lens while the right figure shows the profile of a laser beam that has been shaped with a diffractive beam homogenizer. The right profile clearly shows a brad intensity plateau compared to the left profile.

Typically, the heated area on the water surface has a size of $0.25 \mathrm{~m}^{2}$. For some measurements, where we need a larger elongation of the heated patch in wind direction, we use a second laser with an identical diffractive optical element and place the two square intensity profiles from the lasers next to each other on the water surface. Thereby we can increase the size of the heated area without losing heating power.

If the surface flow is strictly along wind direction, we can reduce the cross-wind elongation of the heated area in order to increase the heat flux. This can be achieved very easily with the use of cylindrical lenses. However, there is an intensity spike in the middle of the heated area, which is due to the $0^{\text {th }}$ diffraction order of the beam homogenizer. This intensity spike becomes a line when cylindrical lenses are used. An example image from a measurement where a cylindrical lens has been used can be seen in figure 4. 


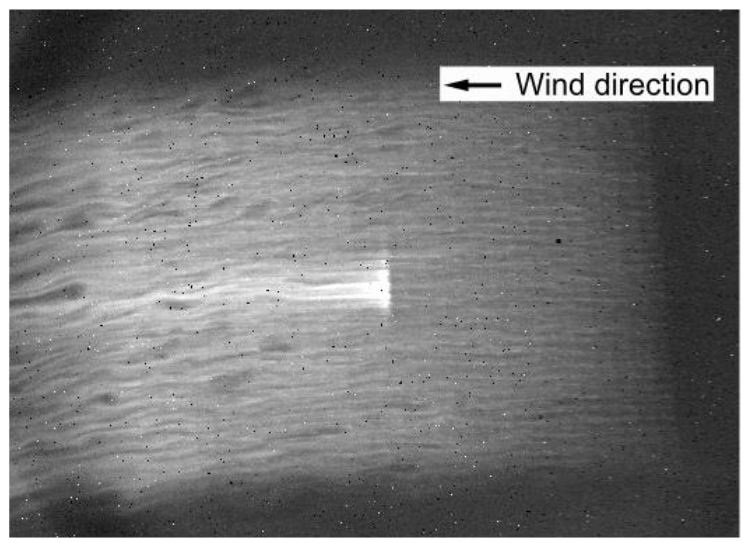

Fig. 4. Example image of a measurement in the Heidelberg wind-wave tank with a reference wind speed of 2.7 $\mathrm{m} / \mathrm{s}$. The temperature difference between the heated area (white) and the unheated area is approximately $0.5 \mathrm{~K}$. The bright slice in the middle of the heated area is due to the $0^{\text {th }}$ diffraction order of the diffractive beam homogenizer that is used to shape the laser beam.

\subsection{Heat excitation scheme}

We apply a lock-in scheme to our active thermography measurements by heating the water surface with different laser on-off frequencies. The air-water interface acts like a low pass filter, enabling the system to reach a thermal equilibrium for low excitation frequencies and damping the temperature amplitudes for higher excitation frequencies. If the laser is turned on and off slowly, the system has enough time to heat up until it reaches a thermal equilibrium. For fast laser on-off frequencies, there is not enough time to reach this equilibrium. We use frequencies up to $25 \mathrm{~Hz}$, which is significantly higher than the $2 \mathrm{~Hz}$ from Jähne et al. [8]. The different heating schemes are visualized in figure 5 .
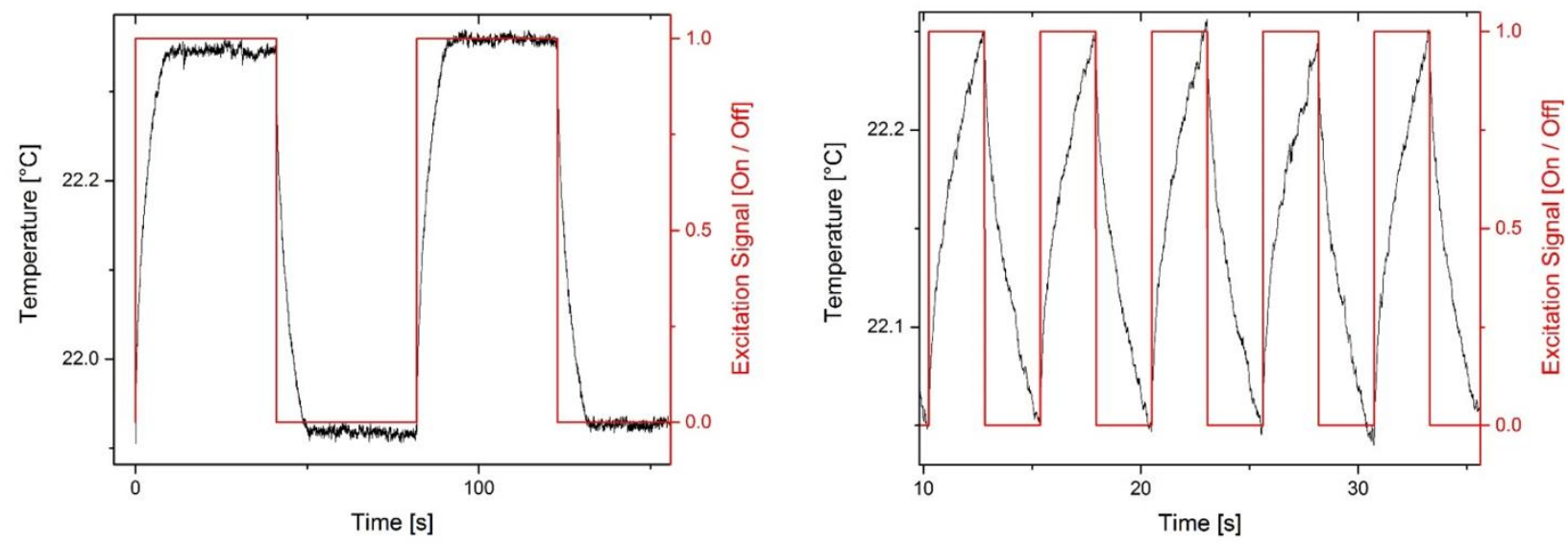

Fig. 5. Illustration of the heating process of the water surface with different laser on-off frequencies $(0.012 \mathrm{~Hz}$ on the left and $0.195 \mathrm{~Hz}$ on the right). With the low frequency, the system has enough time to follow the laser's excitation and to reach a constant temperature, for the higher frequency this is not the case.

The rectangular excitation pattern allows us to use the third and fifth harmonic of the ground excitation frequency for our analysis. In this way we probe the system with three times as many frequencies as compared to a simple sinusoidal excitation pattern. A spectrum of the excitation signal and the measured signal for an excitation frequency of $6.25 \mathrm{~Hz}$ is shown in figure 6 . 

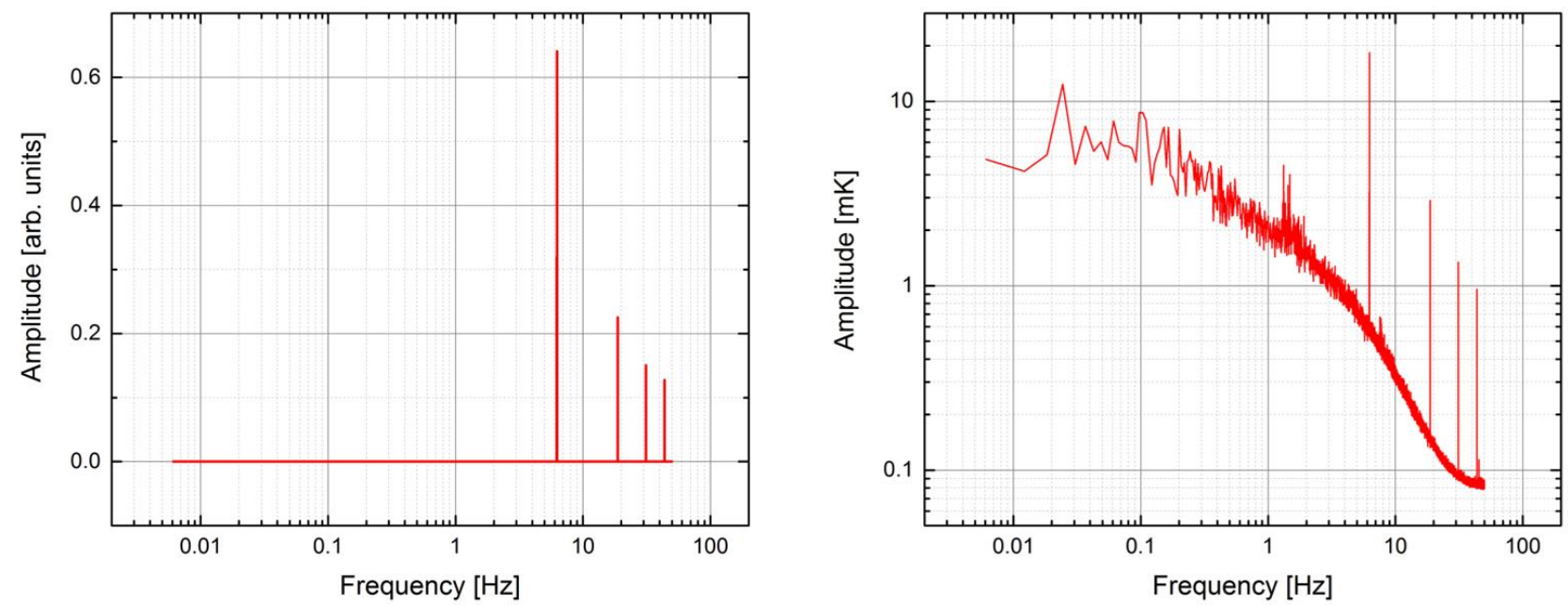

Fig. 6. The left figure shows the spectrum of the excitation signal, that is used by the IR laser to heat the water surface. The base frequency is $6.25 \mathrm{~Hz}$. The $3^{\text {rd }}, 5^{\text {th }}$ and $7^{\text {th }}$ harmonic can clearly be seen. The right figure shows the spectrum obtained from the measured temperature response of the water surface. At the excitation frequencies sharp peaks are detected.

From the analysis in the Fourier domain, we can estimate from which frequency onwards the damping process starts. Figure 7 shows the result of such an analysis.

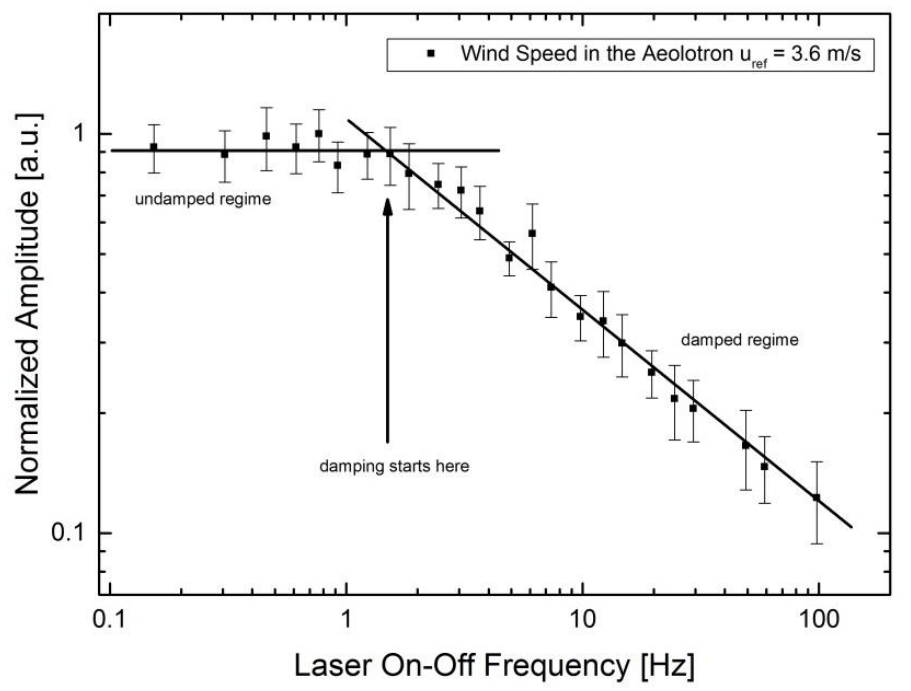

Fig. 7. Example for a frequency analysis from a typical amplitude damping experiment in the Heidelberg windwave tank Aeolotron. When the laser on-off frequency exceeds a certain frequency, the system can't follow the excitation any longer and amplitude damping occurs.

At the frequency $\omega$ where the damping starts, the system has exactly enough time to reach thermal equilibrium, this time corresponds to the settling time $\mathrm{s}$ of the system, i.e.

$$
\omega \tau=1
$$

With the settling time, we can estimate the transfer velocity via the diffusion constant $D$ of heat in water [8]: 


$$
k_{\text {heat }}=\sqrt{\frac{D}{\tau}}
$$

Here it is assumed, that from the frequency where the damping starts onwards only molecular diffusion takes place and turbulent processes no longer play an important role. This justifies the estimation of the transfer velocity by using only the diffusion constant of heat in water.

Figure 8 shows the results obtained with this measurement technique for measurements that have been done in the Heidelberg wind-wave tank Aeolotron. The same measurements were carried out with the old experimental setup using a moving mirror and with the new experimental setup using a diffractive beam homogenizer (see section 2.3 for more details). The use of the diffractive beam homogenizer clearly improves the measurement accuracy.

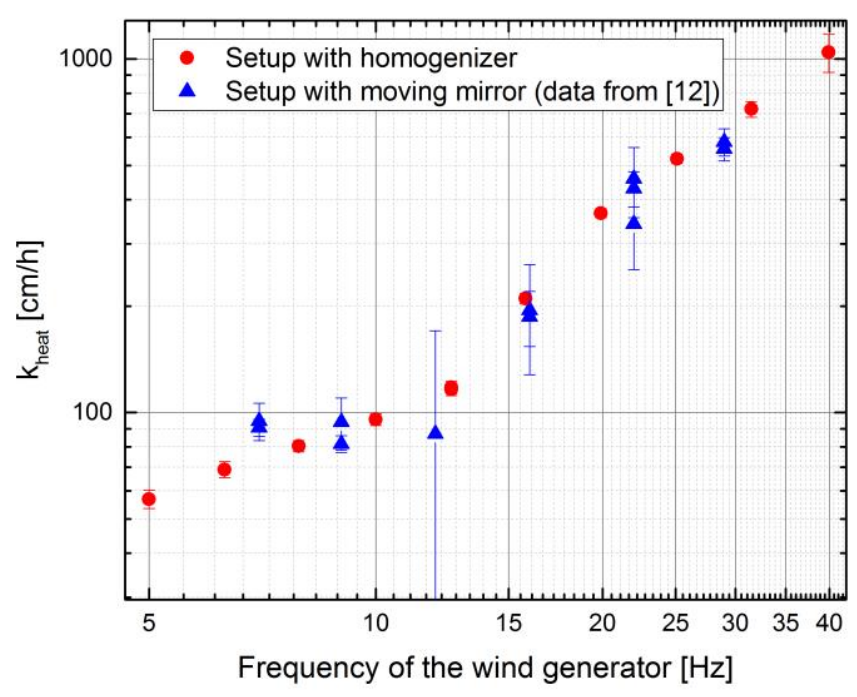

Fig. 8. Comparison of transfer velocities measured with different beam shaping concepts used. The measurement conditions were the same for both measurements and took place in Heidelberg wind-wave tank Aeolotron. It is obvious, that the homogenizer setup has a higher accuracy compared to the moving mirror setup.

\subsubsection{Towards faster measurements}

A typical measurement using the excitation scheme described above takes between 15 minutes and one hour, depending on wind speed. For slower wind speeds, slower frequencies are needed, as the system needs longer to reach thermal equilibrium within the heated area.

This is why we started to use another approach, where we only need two excitation frequencies instead of many different frequencies. In principle we simply use equation 1, but for heat instead of gases. This means that we have to change the concentration difference of the gas in air and water into the energy difference due to the temperature difference $\Delta T$ at the water surface, the density $\rho$ and the heat capacity $c_{p}$ of water:

$$
k_{\text {heat }}=\frac{j_{\text {heat }}}{\rho c_{p} \Delta T}
$$

The heat flux jheat is theoretically given by the output power of the laser and the size of the heated area. However, the optical elements are not perfect and this reduces the actual heat flux. Thus we estimate the heat flux at the water surface from the measurement instead of using theoretical values. To estimate the heat flux, we need a high excitation frequency, because then we are in a regime, where only molecular diffusion is important and we can estimate the heat flux from the measured temperature amplitude at the excitation frequency $\omega[8]$ :

$$
j_{\text {heat }}=\rho c_{p} \Delta T \sqrt{D \omega}
$$




\subsection{1/qirt.2016.069}

To apply formula 5 only the temperature difference in thermal equilibrium is missing. We get this temperature difference by applying a very low excitation frequency. In principle we could keep the laser on constantly, but by using a very low frequency, it is easier to filter the temperature increase from the constant offset signal when applying FFT. The frequency we use for this is so small, that a water element can travel through the heated area completely within half of the periodic time of the excitation frequency.

As we only need two frequencies, these measurements are much faster and can be done within approximately five minutes. However, we lose measurement accuracy with this approach.

A comparison between the two different approaches to get heat transfer velocities from active thermography is shown in figure 9. It becomes obvious, that both methods are in good agreement, deviating only as little as four percent from each other. It can clearly be seen from figure 9 that the faster measurement scheme, which is labelled " $\Delta T$ method" comes at the cost of a reduced measurement accuracy. However, a higher accuracy might be misleading. The data of figure 9 was taken under laboratory conditions with constant wind speeds and controlled boundary conditions. For field measurements on the ocean, it might be more important to measure faster, as the boundary conditions might change quickly. Otherwise an averaging over different conditions would occur and weaken the significance of the measurement. So for field measurements the faster measurement might even lead to more precise results.

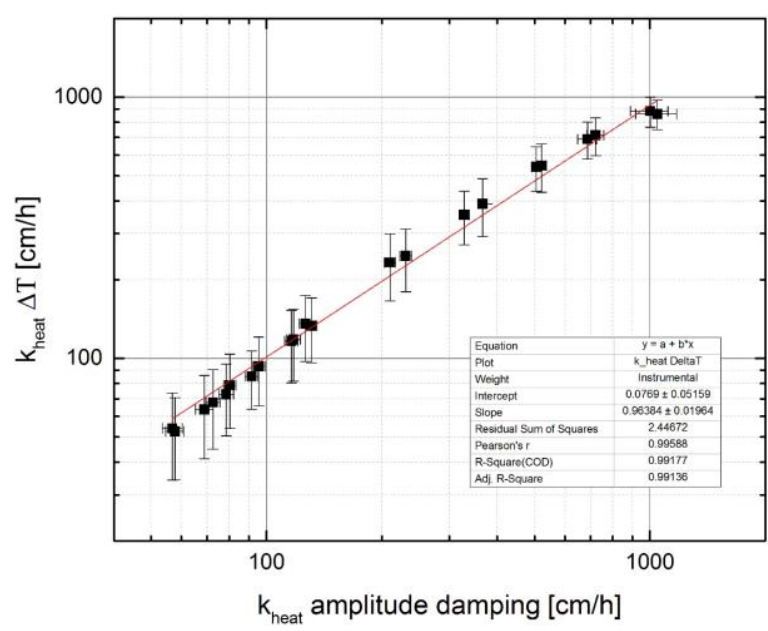

Fig. 9. Comparison for the amplitude damping method and the $\Delta T$ method for the analysis of experiments with clean water and different wind speeds in the Heidelberg wind-wave tank Aeolotron.

To avoid the use of two successive measurements with different frequencies we are currently implementing another excitation scheme, where we convolute two excitation spectra. This allows for the simultaneous measurement of the heat flux and the temperature difference in equilibrium. The basic idea of this convolution of spectra, which corresponds to a multiplication of two rectangular signals in the time domain, is shown in figure 10 .

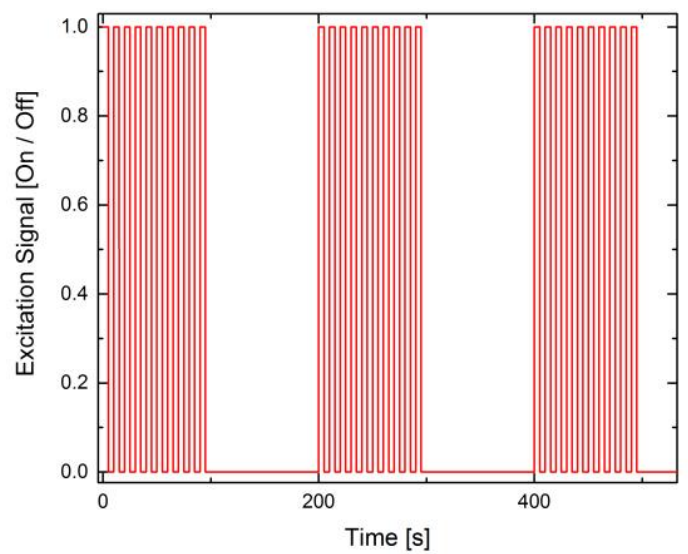

Fig. 10. Example for an excitation scheme for a faster measurement. The slow and fast frequency spectra are convoluted, which corresponds to a multiplication of the two rectangular signals in the time domain. Thus both frequencies can be measured at the same time. 


\subsection{1/qirt.2016.069}

By looking at figure 10 it becomes obvious, that the temperature amplitude of the low frequency will drop by a certain factor, that depends on the chosen duty cycle of the excitation frequency. The same is true for the high frequency. Optimizing the duty cycle is thus important in order to be able to deposit enough energy onto the water surface to be able to measure temperature changes with the IR camera accurately.

\section{Conclusion}

This contribution has presented thermography as a very effective tool for the investigation of air-sea heat and also air-sea gas exchange. The high-resolution, thermally and temporally, exceeds any other measurement technique for the air-water gas transfer velocity. The new multifrequency approach allows for unbiased fast field measurements. Compared to the many frequency stimulation method, the measuring time is reduced from about 30 minutes to 5 minutes.

\section{REFERENCES}

[1] Wanninkhof R., Asher W.E., Wepperning R., et al., Gas transfer experiment on georges bank using two volatile deliberate tracers, J GeophysRes 98(C11):20237-20248, 1993, DOI: 10.1029/93JC01844

[2] Watson A.J., Upstill-Goddard R.C., Liss P.S., Air-sea exchange in rough and stormy seas measured by a dual tracer technique. Nature 349(6305):145-147, 1991, DOI: 10.1038/349145a0

[3] Roether W., Kromer B., Optimum application of the radon deficit method to obtain air-sea gas exchange rates, Gas transfer at water surfaces. Reidel, Hingham, pp 447-457, 1984

[4] Broecker W.S., Peng T.-H., Ostlund G., et al., The distribution of bomb radiocarbon in the ocean, J Geophys Res 90:6953-6970, 1985, DOI: 10.1029/JC090iC04p06953

[5] Garbe C.S., Spies H., Jähne B., Estimation of complex motion from thermographic image sequences, Proc. SPIE 5073, Thermosense XXV, 303, 2003, DOI:10.1117/12.501121

[6] Kräuter C., Visualization of air-water gas exchange, PhD thesis, Heidelberg University, 2015

[7] Kräuter C., et al., High resolution 2-D fluorescence imaging of the mass boundary layer thickness at free water surfaces, J. Europ. Opt. Soc. Rap. Public. 9 14016, 2014, DOI: 10.2971/jeos.2014.14016

[8] Jähne, B., Libner P., Fischer R., et al., Investigating the transfer process across the free aqueous boundary layer by the controlled flux method, Tellus B, 41B, 177-195, 1989, DOI: 10.1111/j.1600-0889.1989.tb00135.x

[9] Haußecker H., Heat as a proxy tracer for gas exchange measurements in the field: principles and technical realization, Air-Water Gas Transfer: Selected Papers from the Third International Symposium on Air-Water Gas Transfer, AEON, 405-413, 1995, DOI: 10.5281/zenodo.10401

[10] Haußecker H., In situ measurements oft he air-sea gas transfer rate during the MBL/CoOP west coast experiment, Air-Water Gas Transfer: Selected Papers from the Third International Symposium on Air-Water Gas Transfer, AEON, 775-784, 1995, DOI: 10.5281/zenodo.10407

[11] Schimpf U., Garbe C., and Jähne B., Investigation of transport processes across the sea surface microlayer by infrared imagery, J. Geophys. Res., 109, C08S13, 2004, DOI: 10.1029/2003JC001803

[12] Garbe C. S., Schimpf U., and Jähne B., A surface renewal model to analyze infrared image sequences of the ocean surface for the study of air-sea heat and gas exchange, J. Geophys. Res., 109, C08S15, 2004, DOI: $10.1029 / 2003 J C 001802$

[13] Nagel L. et al., Comparative heat and gas exchange measurements in the Heidelberg Aeolotron, a large annular wind-wave tank, Ocean Sci., 11, 111-120, 2015, DOI:10.5194/os-11-111-2015

[14] Asher W. E., Jessup A. T., Atmane M. A., Oceanic application of the active controlled flux technique for measuring air-sea transfer velocities of heat and gases, J. Geophys. Res., 109, C08S12, 2004, DOI: 10.1029/2003JC001862 